\title{
Spread of Local Transmission Virus That Causes Health Problems From Public Transportation
}

\author{
Cadeo Canh Bao ${ }^{1}$, Due Bihn ${ }^{1}$ \\ ${ }^{1}$ Hanoi University of Public Health, Vietnam \\ Received: December 5, $2021 \quad$ Received in Revised: January 7, $2021 \quad$ Accepted: January 17, 2021
}

\begin{abstract}
Public transportation and infrastructure are particularly prone to becoming a hub for the spread of Covid-19. It is a challenge that must be overcome due to the high mobility of people who use public transit. The Corona virus is primarily disseminated via droplets when liquid drops out and enters the mouth, nose, or eyes. Covid-19 droplets, on the other hand, can adhere to the surface of objects for an extended period of time. The virus is spreading more rapidly via local transmission. In other nations, 90 percent of those infected with Omicron appear to be asymptomatic at the moment. Or if the symptoms are mild to severe in character. For instance, influenza, a general malaise, a mild fever, a mild cough, and painful swallowing. As a result, it poses a significant hazard to public health.
\end{abstract}

Keywords: Local Transmission, Virus, Public Transportation

\section{Introduction}

COVID-19 infection is frequently associated with symptoms of acute respiratory distress such as fever, coughing, and shortness of breath. The typical duration of incubation is 5-6 days, whereas the greatest duration is 14 days. COVID-19 can cause pneumonia, acute respiratory syndrome, renal failure, and even death in extreme instances. The majority of individuals had a temperature, with some experiencing trouble breathing, and X-rays revealed significant pneumonia infiltrates in both lungs. COVID-19 is spread from person to person by coughing/sneezing droplets, not via the air. Individuals in close touch with COVID-19 patients, particularly those who care for COVID-19 patients, are most at risk of developing this disease. The standard suggestions for preventing infection are to wash hands often with soap and clean water, to practice proper coughing and sneezing etiquette, to avoid direct contact with livestock and wild animals, and to avoid close contact with anybody who is coughing or sneezing. Additionally, infection prevention and control measures should be implemented in health care settings, particularly emergency rooms (World Health Organization (2020). Additionally, because the corona virus is transmitted mostly through human to human contact, the spread becomes more aggressive. Certain instances associated with transmission from asymptomatic carriers typically involve close interaction with COVID-19 patients. Numerous other factors have been proven, including the propagation of the corona virus via droplets, the air, fomites, and faecal-oral transmission, blood, mother-to-child transmission, and animal-to-human transmission, all of which are currently in the research stage.

Contact-based transmission can be classified as direct contact, indirect contact, or close contact with persons infected with the corona virus by saliva production or respiratory tract secretions known as droplets. Droplets are classified into two types: respiratory droplets with a diameter more than or equal to $5 \mathrm{~m}$ and droplet nuclei or aerosols with a diameter greater than or equal to $5 \mathrm{~m}$ (Zhao et al., 2020). When a person is infected, respiratory droplets from the sick person might infect other persons in close proximity. With local transmission, Omicron's spread is 
jeopardized. Omicron is a corona virus variation that spreads more quickly than other variants but does not produce more severe illness (He et al., 2021). Public transportation and infrastructure are particularly prone to become a hub for the spread of Covid-19. Due of the great mobility of individuals who use public transit, this is a significant issue that must be addressed.

\section{Transmission of Covid-19 Infects Humans}

COVID-19 patients typically have a temperature of 380C, a dry cough, and shortness of breath. If a person has been to an infected nation or has cared for/close contact with a person who has COVID-19 within 14 days of exhibiting these symptoms, that person will be submitted to further laboratory testing to confirm the diagnosis.

COVID-19, like other respiratory viruses, can cause moderate symptoms such as a runny nose, sore throat, cough, and fever. Around $80 \%$ of cases resolve spontaneously. Around one in every six people will acquire a serious disease, such as pneumonia or difficulties breathing, which will often progress gradually. Although this illness has a low fatality rate (about 3\%), older adults and those with pre-existing medical disorders (such as diabetes, high blood pressure, or heart disease) are often more susceptible to falling unwell. In light of recent breakthroughs, more than half of confirmed cases have been deemed to be improving, and the cure rate is expected to continue to rise.

Currently, human-to-human transmission of SARS-CoV-2 is the primary mode of infection, resulting in a more aggressive spread (Khan et al., 2020; Arslan et al., 2020). Dhand \& Li (2020) indicate Transmission of SARS-CoV-2 from symptomatic individuals occurs via droplets emitted from the nose or mouth during coughing or sneezing. The droplets then settle on nearby objects. Subsequently, if another person touches an object contaminated with these droplets and then contacts their eyes, nose, or mouth, they can get COVID19. Alternatively, someone might become infected with COVID-19 after inhaling droplets from an infected individual. This is why it is critical for us to keep a minimum of one meter apart from ill people. Additionally, SARS-CoV-2 has been shown to remain viable in aerosols (produced by a nebulizer) for at least three hours. WHO estimates COVID-19's reproductive number (R0) to be between 1.4 and 2.5. Other research, on the other hand, indicate a R0 of 3.28.

According to the WHO, the chance of transmission from someone who does not exhibit symptoms of COVID-19 is extremely improbable at the moment. However, many patients who are diagnosed with COVID-19 have very modest symptoms such as a slight cough and do not complain of discomfort, which may occur during the early stages of the disease. Until recently, specialists have been conducting research to ascertain the transmission or incubation time of COVID-19. Multiple experiments done demonstrate that SARS-CoV-2 is more stable on plastic and stainless steel materials ( $>72$ hours) than on copper ( $>4$ hours) and on cabinets up to the ventilation fan.

Indirect viral transmission occurs when a person comes into contact with infected surfaces in their immediate environment (Stadnytskyi et al., 2021). Transmission via inanimate things is more severe if they come into direct contact with the lips, eyes, or nose. Several recent but unconfirmed discoveries claimed the detection of SARS-CoV-2 RNA in biological materials such as feces, urine, serum, or while speaking, singing, coughing, or sneezing.

\section{Spread of Transmission Virus through Public Transportation}

The number of Covid-19 increases has grown significantly during the previous week. Disinfectant spraying on public transit, as this is considered a possible area for the spread of 
Covid 19. Disinfectant liquid spraying on public transit, This action aims to restrict the transmission of Covid 19, a virus that is prevalent in public transportation. The spraying protects the public or users of public transportation from the spread of the Corona Virus.

Put on a mask, maintain a safe distance, and wash your hands thoroughly with soap and running water. By intensifying $3 \mathrm{M}$ socialization, it is intended that the public would become more aware and worried about the importance of wearing masks and maintaining a safe distance on public transit. The community is required to wear masks when participating in activities outside the house. Additionally, Public transportation drivers must restrict passengers to $50 \%$.

To far, COVID-19 instances of the Omicron variation have been reported in 150 countries worldwide, the majority of which have infected various industrialized nations till reaching a peak and exceeding the preceding wave, namely the Delta version. The majority of the increase in Covid-19 instances is due to international travel (Russell et al., 2021). This growth also has a direct effect on hospital treatment, particularly in the United States and Australia, as well as the United Kingdom and Europe. Another upward trend was observed in India, the Philippines, and Japan, while a large decline in incidence was observed in South Africa. The public has been urged not to travel overseas in the coming weeks in order to avoid the spread of the corona virus. Cases of travelers dominated the daily case count, resulting in an increase in active cases and patient care. On January 9, for example over 300 of the 393 instances were caused by foreign tourists. Thus, we once again implore our friends to abstain from traveling overseas unless absolutely necessary. Additionally, the government has been paying close attention to the travelers policy since the Christmas and New Year's vacations, which might result in the transmission of the Omicron variety. The travelers of this evidence have returned several times with a great deal of difficulty regarding this Omicron (Abbasi, 2021). Entrance security measures will be maintained in order to prevent the arrival of the Omicron variety, which will quickly spread across the community. The positive case rate, or the Covid-19 positivity rate, for international arrivals in the country reached $13 \%$, while the positive case rate for domestic transmission was 0.2 percent. The positivity rate for international arrivals is 65 times that of domestic transmissions. This reinforces our notion that the majority of positive cases now happening are the result of international immigration.

\section{Potential for widespread local transmission of the Omicron variant virus}

Meanwhile, Griffith University Australia's Center for Environmental and Population Health, stated that it is extremely likely that local transmission instances will continue to spread throughout wander the population. The reason for this is because Omicron patients are generally asymptomatic. This is in reference to the two instances discovered in Surabaya that had recently arrived.

What is occurring is referred to as scientific presumption, and it refers to what is occurring in other nations, where it appears that 90 percent of those infected with Omicron are now asymptomatic. Or if the symptoms are mild to severe in character. For instance, influenza, a general malaise, a mild temperature, a mild cough, and difficulty swallowing. Indeed, these are emotions that people experience practically every year. By 2022, there is a risk of Omicron instances spreading. Asymptomatic does not indicate healthy or incapable of transmission. However, this has the ability to last a long time Covid-19 and will affect the survivors' quality of life and health by $10 \%$ to $30 \%$. The increase of Covid-19 cases and the development of local transmission of the Omicron variant are expected to reach a zenith around late February or early March 2022. As a result, limits on community activities are implemented in accordance with immunization and health protocol requirements. Individuals are once again advised to minimize their movement. The daily surge of Covid-19 instances prompted a small online conference to 
explore the installation of community activity limitations. Daily case additions increased to 1,054 from 850 the previous day. Case additions decreased to 855 cases. At the moment, more cases of the Omicron variety are produced through indigenous transmission than by international tourists. Almost entirely, more than 90\%, local transmission.

\section{Conclusion}

COVID-19 infection is frequently associated with symptoms of acute respiratory distress such as fever, coughing, and shortness of breath. The typical duration of incubation is 5-6 days, whereas the greatest duration is 14 days. Infection prevention and control measures should be implemented in health care settings, particularly emergency rooms. COVID-19 patients typically have a temperature of $380 \mathrm{C}$, a dry cough, and shortness of breath. Around $80 \%$ of cases resolve spontaneously. Around one in every six people will acquire a serious disease, such as pneumonia or difficulties breathing, which will often progress gradually. SARS-CoV2 RNA is more stable on plastic and stainless steel materials than on copper. The community is required to wear masks when participating in activities outside the house. Disinfectant spraying on public transit, as this is considered a possible area for the spread of Covid 19. Over 300 of the 393 instances were caused by foreign tourists. The positive case rate for international arrivals in the country reached $13 \%$, while the positivity rate for domestic transmissions was $0.2 \%$. By 2022, there is a risk of Omicron instances spreading. Asymptomatic does not indicate healthy or incapable of transmission. However, this has the ability to last a long time Covid-19 and will affect the survivors' quality of life.

\section{References}

Abbasi, J. (2021). Omicron Has Reached the US-Here's What Infectious Disease Experts Know About the Variant. Jama, 326(24), 2460-2462.

Arslan, M., Xu, B., \& El-Din, M. G. (2020). Transmission of SARS-CoV-2 via fecal-oral and aerosols-borne routes: Environmental dynamics and implications for wastewater management in underprivileged societies. Science of the Total Environment, 743, 140709.

Dhand, R., \& Li, J. (2020). Coughs and sneezes: their role in transmission of respiratory viral infections, including SARS-CoV-2. American journal of respiratory and critical care medicine, 202(5), 651-659.

He, X., Hong, W., Pan, X., Lu, G., \& Wei, X. (2021). SARS-CoV-2 Omicron variant: characteristics and prevention. MedComm.

Khan, S., Liu, J., \& Xue, M. (2020). Transmission of SARS-CoV-2, required developments in research and associated public health concerns. Frontiers in Medicine, 7, 310.

Russell, T. W., Wu, J. T., Clifford, S., Edmunds, W. J., Kucharski, A. J., \& Jit, M. (2021). Effect of internationally imported cases on internal spread of COVID-19: a mathematical modelling study. The Lancet Public Health, 6(1), e12-e20.

Stadnytskyi, V., Anfinrud, P., \& Bax, A. (2021). Breathing, speaking, coughing or sneezing: What drives transmission of SARS-CoV-2?. Journal of Internal Medicine.

World Health Organization. (2020). Infection prevention and control during health care when COVID-19 is suspected: interim guidance, 19 March 2020 (No. WHO/2019nCoV/IPC/2020.3). World Health Organization. 
Zhao, L., Qi, Y., Luzzatto-Fegiz, P., Cui, Y., \& Zhu, Y. (2020). COVID-19: effects of environmental conditions on the propagation of respiratory droplets. Nano letters, 20(10), 7744-7750. 